\title{
The effects of isolation rearing on exploration in the rat
}

\author{
B. J. SAHAKIAN, T. W. ROBBINS, and S. D. IVERSEN \\ University of Cambridge, Cambridge, England
}

\begin{abstract}
The hypothesis that isolation rearing enhances exploration was tested in two settings which varied the extent to which exploratory behavior would be affected by competing hyperactivity. Experiment 1 measured exploration as contact of a discrete novel stimulus, in terms of bout frequency and duration. Locomotor activity was measured by photocell beam interruption. Isolation-reared rats were hyperactive, showed an increased incidence of exploratory bouts but no differences in duration of exploratory behavior, compared with group-reared controls. Experiment 2 measured, independently, locomotor activity and the preference for a novel environment over a familiar one. Isolation-reared rats, whether male or female, showed enhanced novelty preference compared with controls. No significant differences were found in locomotor activity. The results are discussed in terms of the hyperactivity of isolates interfering with investigative behavior by response incompatibility.
\end{abstract}

Rearing conditions are crucial determinants of adult behavior and brain development (Rosenzweig, 1971; Valzelli, 1973). Rats reared in isolation from weaning show several behavioral abnormalities. For example, they are more active in the open field (Einon, Morgan, \& Sahakian, 1976; Mandell, Segal, Kuczenski, \& Knapp, 1973; Morgan, 1973; Syme, 1973) and in photocell activity cages (Sahakian, Robbins, Morgan, \& Iversen, 1975). Isolates also show evidence of retarded habituation, compared with group-reared animals (Einon et al., 1976; Sahakian et al., 1975).

It is not clear whether the hyperactivity of isolates is associated with an enhanced tendency to explore the environment, as there have been discrepancies in the literature (Lore \& Levowitz, 1966; Luchins \& Forgus, 1955). The contradictory reports arise from two main sources: differences in method and time of isolation and differences in both definition and measurement of exploration. In particular, certain measures of exploration, such as latency to emerge (Lore \& Levowitz, 1966), are confounded with emotional factors, whereas others are confounded with locomotor activity (Berlyne, 1960; Leyland, Robbins, \& Iversen, 1976; Welker, 1957).

In these experiments, rats were reared in isolation from weaning. Exploration was measured in two distinct settings, which, however, had certain features in common. In both, exploration was defined as

Thanks are due to Dr. R. Miller for his comments on an earlier draft, to Professor O. L. Zangwill for research facilities, and Dr. D. F. Einon for supplying us with the male rats used in Experiment 2. B.J.S. is in receipt of a Cambridge Philosophical Society Studentship. The authors' address is: Psychological Laboratory, Downing Street, Cambridge, CB2 3EB, England. behavior directed towards novel stimuli, since it is generally accepted that novelty is a major determinant of exploration (Berlyne, 1960; Schneider $\&$ Gross, 1965). Animals were familiarized to the test situation prior to the introduction of novel stimuli, in order to reduce emotional responses elicited by a novel test situation and to assess more precisely behavior directed towards particular novel stimuli.

Since isolates are hyperactive compared with controls, it is possible that any measure of exploration which is based on the duration of physical contact with a discrete novel stimulus ("inspective exploration," Berlyne, 1960) will show the isolates to be less exploratory than controls. Investigatory responses directed toward the stimulus will tend to be in competition with high levels of locomotor activity. Therefore, in this study, the two settings employed varied the extent to which the measure of exploration would be dependent on competing levels of locomotor activity. In Experiment 1, an apparatus (Berlyne, 1955) was employed which measures exploration in terms of duration and bout frequency of contact with a discrete novel object. In Experiment 2, preference for a novel over a familiar environment was measured in a setting that permitted an independent measure of activity. In the latter situation, it is possible for an animal to be hyperactive and yet show an enhanced preference for novelty. A comparison of results found in the two experiments might clarify the effects of isolationrearing on exploratory behavior.

\section{EXPERIMENT 1}

\footnotetext{
Method

Subjects. The subjects for these experiments were 24 female
} 
hooded rats aged 60-70 days at time of testing. These were randomly selected from a larger population that were purchased from Animal Suppliers Ltd. in litters with their mothers at $13 \pm 1$ ) days of age. All animals were weaned at 17 days and housed (cages, $15.5 \times 15.5 \times 23 \mathrm{~cm}$ ) either individually $(\mathrm{n}=12$ ) or in three groups of four rats per cage $(41 \times 25 \times 20 \mathrm{~cm})$. Food and water was supplied ad lib and the rats were placed on a 12-h light-dark cycle $(0800-2000 \mathrm{~h}$ light). All rats were housed in the same room, and thus could hear and smell other rats.

Apparatus. A full description of the "Berlyne box" can be found in Robbins and Iversen (1973). Briefly, the box consists of an open field $(61 \times 45.6 \times 30.4 \mathrm{~cm})$ with three photocells distributed evenly along the $61-\mathrm{cm}$ side of the apparatus at intervals of approximately $15 \mathrm{~cm}$, and with an adjoining alley $(20.3$ $\times 10.1 \times 30.4 \mathrm{~cm}$ ) at one end. A photocell (photocell beam 1) was located at the entrance to the alley.

Two objects, an old wool glove on a bottle and a spool of wire with a scrub brush stuck in it, were used to elicit exploration. These objects, which were similar in size, fitted securely into the end of the alley furthest from the open field, and measured approximately three-quarters the height of the alley. Preliminary tests had established that these test objects were potent elicitors of exploratory behavior.

A modification of the Berlyne box was the addition of a Perspex lid, allowing video recording of the experiment. The video system was especially sensitive to low-level illumination, allowing the apparatus to be evenly and dimly lit. Video recordings of the alley and surrounding area and a superimposed automatic timer provided temporal resolution of $0.1 \mathrm{sec}$, enabling exact measurements of duration of active object exploration to be made. All recording apparatus was located in a separate room.

Procedure. The 24 subjects were divided into four groups. Six isolation-reared rats were exposed to the same test object on 2 successive days; six group-reared rats were exposed to the same object on 2 successive days; six isolation-reared rats were exposed to a different object on 2 successive days; and finally, six groupreared rats were exposed to a different object on 2 successive days.

On Day 1, each rat was habituated to the empty Berlyne box for $1 \mathrm{~h}$ and activity readings from each of the photocells were taken every $10 \mathrm{~min}$. The rat was then returned to its home cage for $5 \mathrm{~min}$ while an object was placed at the end of the alley. Then the rat was replaced in the open field of the Berlyne box and tested for frequency and duration of active exploration over a 10-min period. Exploration was defined as occurring when the animal sniffed or contacted the test object with any part of its head or forelimbs. This classification included, for example, rearing at the object.

Both duration and frequency (or "bouts") of exploration and locomotor activity were recorded on each 2 -min period of the 10-min trial. The rat was then returned to its home cage, and the box was sprayed with a mild deodorant to mask odors before testing of the next rat.

On Day 2, the retest trial, the procedure was identical to that of Day 1, except that half of the rats were exposed to the same object as on Day 1, while the other 12 rats were exposed to a different object. The objects were counterbalanced across same and different conditions. The testing order of the animals was also counterbalanced, with all testing being conducted in the afternoons $(1400-1800 \mathrm{~h})$.

The video recordings were later analyzed for exact measurements of frequency and duration of exploration. Interobserver correlation coefficients were all $\geqslant 0.850$ for these measurements.

\section{Results}

All analyses followed the same basic model with the two factors Same vs. Different Type Objects and Rearing Condition being orthogonal to the other factors, which were Days ( 1 or 2 ) and Time Course. In the analyses of locomotor activity, the distribution of activity across the four photocell beams was also taken into account.

Habituation activity. As shown in Figure 1, isolation-reared animals were significantly more active than controls $[F(1,20)=32.32, p<.001]$ during the habituation periods of both days. There was a significant decline in activity over the $60 \mathrm{~min}$ $[F(5,100)=50.43, p<.001]$. There was also a significant difference in the activity registered by the four photocell beams $[F(3,60)=10.97, p<.001]$, with activity being highest at photocell beam 2 (Figure 2).

Test activity. The isolation-reared animals showed significantly higher levels of locomotor activity than controls $[\mathrm{F}(1,20)=12.30, \mathrm{p}<.01]$. Activity declined significantly during the course of both days $[F(4,80)=13.06, p<.01$, Figure 1$]$, and it also differed over the four photocell beams $[F(3,60)=$ $114.76, \mathrm{p}<.001$, Figure 2]. The presence of stimulus objects in the alley greatly changed the distribution of activity within the apparatus. The isolation-reared animals showed a different distribution of activity from that of controls $[F(3,60)=2.99, p<.05]$.

Exploration duration. As the distribution of the duration data was strongly positively skewed, they were subjected to a logarithmic transformation. Analysis of variance revealed no significant main effects for any variable. However, the interaction among same vs. different test objects, rearing condition, and days was significant $[F(1,20)=4.74$, $\mathrm{p}<.05$ ]. As can be seen from Figure 3, this interaction resulted from the diminution of exploration in the group-reared animals which occurred to the same stimulus object on Day 2. No such decrement was observable for isolation-reared animals. This suggests that the isolation-reared animals showed less habituation towards the test object introduced on Day 1 when the same object was present during Day 2 than did group-reared animals. However, exploration of a different object on Day 2 showed only a small decline in both groups.

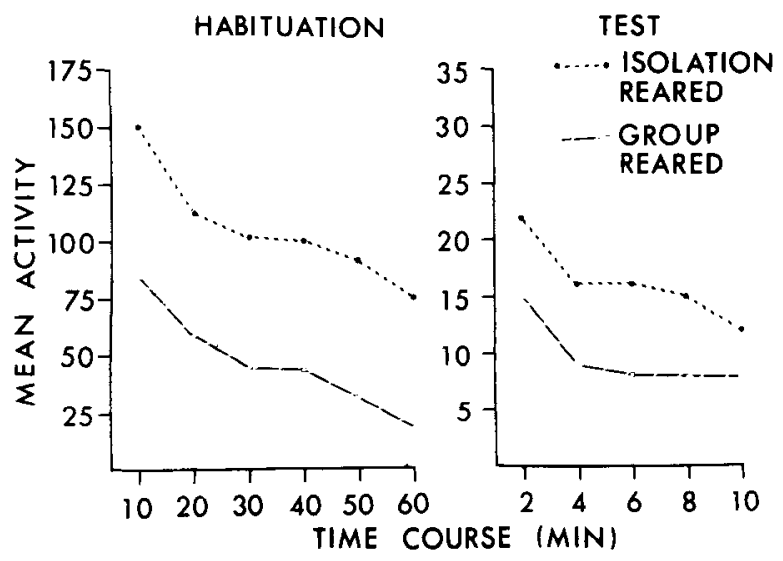

Figure 1. Locomotor activity during the habituation trial and the test trial of isolation-reared and group-reared rats in Experiment 1. Data shown are combined over Days 1 and 2. 


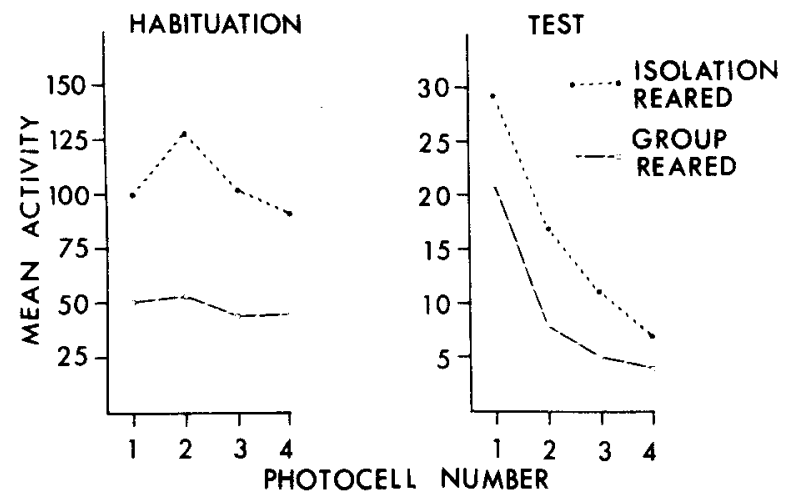

Figure 2. Locomotor activity over the four photocell beams for the habituation and the test trial of isolation-reared and groupreared rats in Experiment 1. Data are combined over Days 1 and 2.

It should also be noted that the level of exploratory behavior maintained by the test objects was very high (mean $=412.5 \mathrm{sec}$ ), taking up $68.8 \%$ of the total trial length.

Bouts of exploration. Isolated animals showed a significantly higher frequency of bouts of exploration than did controls $[F(1,20)=9.33, p<.01]$. The mean frequency of bouts for isolated and grouped rats were 5.77 and 4.42 bouts/ 2 -min period, respectively. Overall, the frequency of bouts declined over the time course of the trial from a mean of 8.27 in the first $2 \mathrm{~min}$ to a mean of 3.73 in the last $2 \mathrm{~min}$ of the 10 -min trial.

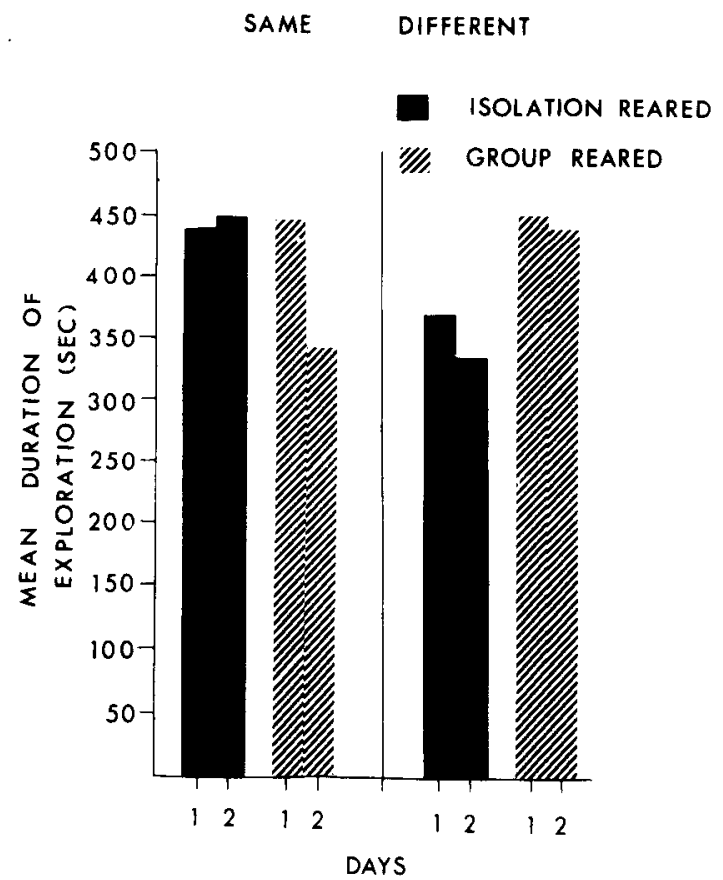

Figure 3. Mean duration of exploration (seconds) in the two test-object conditions (same and different) of isolation-reared and group-reared rats in Experiment 1.

\section{Discussion}

No differences occurred between isolation- and group-reared rats in duration of exploration, but the isolates had a significantly higher frequency of bouts of exploration. Isolates therefore initiated exploratory sequences of behavior more often than controls, but terminated them sooner. The isolates were also significantly more active than controls in the main compartment of the apparatus.

It is difficult to compare these results with other experiments, since the most similar of these have studied the effects of isolation on exploration of a number of different stimuli in an open field with no prior habituation. Lore and Levowitz (1966) found that isolated rats showed more object-contact exploration than controls, although Einon and Morgan (1976) attributed this result to retarded habituation of exploratory activity in isolates rather than to an enhanced initial level. The latter interpretation is partially supported by the present results in that isolates showed enhanced exploration of objects retained from a previous trial (same condition, Figure 3), compared with group-reared animals. However, despite the evident intratrial habituation of locomotor activity, there was no parallel tendency to show intratrial habituation of exploration. This was possibly because of the effectiveness of the stimulus objects in eliciting exploration; $68.8 \%$ of the total trial length was occupied with exploratory behavior. The use of a prior habituation period, which enhanced the novelty of the exploranda relative to the rest of the test situation, probably contributed to this greater efficacy.

Exploratory behavior directed towards the novel stimulus, restricted to the alley of the Berlyne box, is incompatible with activity in the main portion of the apparatus. It would then be expected that high levels of locomotor activity would tend to interfere with high levels of investigatory behavior directed towards the object ("inspective exploration," Berlyne, 1960). Indeed, d-amphetamine, a drug which increases locomotor activity at certain doses, decreases the duration of exploratory behavior (Robbins \& Iversen, 1973). Therefore, the higher frequency of bouts of exploration in the isolates may represent an increased exploratory tendency. However, the maintenance of investigative behavior of a particular stimulus is disrupted in isolates by their hyperactivity. This interpretation is supported by the fact that isolates would often interrupt a bout of exploration with a very fast burst of activity in the main compartment, apparently unrelated to the investigation of the environment. Therefore, it is possible that the measure of exploration based on the duration of contact of a stimulus object in Experiment 1 is relatively insensitive for detecting differences between isolation- and group-reared rats. 


\section{EXPERIMENT 2}

Experiment 2 utilized an "exploration choicebox" to test the hypothesis that a test setting measuring exploration in terms of novelty preference, independent of locomotor activity, might find isolates to be more exploratory than controls. Following a 1-h habituation period to one half of a large box, rats were given a choice between that side and the other identical, but not previously experienced (novel), side. Measures of exploration and activity were employed that were independent of each other. Therefore, the animals could show evidence of an exploratory tendency while simultaneously being active over a wide area. For example, the present technique has been used to show that methylphenidate enhances locomotor activity while simultaneously producing an elevated preference for a familiar environment (Dyne \& Hughes, 1970).

\section{Method}

Subjects. The subjects were 20 female hooded rats randomly selected from the same population of rats used in Experiment 1. Ten rats had been isolation-reared and 10 were group-reared. Another population of male hooded rats was used in a partial replication of the experiment. These had been purchased from Animal Suppliers Ltd. at 13 days of age, with their mothers, and weaned at 16 days of age. At this time, half of the rats were reared in isolation (cages, $38 \times 2619 \mathrm{~cm}$ ) and the other half reared in groups of 5 (cages, $41 \times 25 \times 20$ ). Again, 10 male rats had been isolation-reared and 10 were group-reared. The slight differences in rearing experience between male and female rats in Experiment 2 has not been found to be an important variable in other experiments carried out in this laboratory. The male rats were tested at 60-70 days of age.

Apparatus. The apparatus was a large rectangular box $(118 \times$ $43.5 \times 29 \mathrm{~cm}$ ) with three aluminum sides and a transparent Perspex front. Attached to the center of the Perspex front was a Perspex startbox $(9 \times 15 \times 15 \mathrm{~cm})$, which was not used in this experiment. The apparatus could be separated into two halves $(59 \times 43.5 \times 29 \mathrm{~cm})$ by an aluminum divider. The floor was made of two wire mesh grid floors which fitted on either side of the aluminum divider. An aluminum tray beneath the grids served to collect the excrement. On the tray, a line of black tape running perpendicular to the aluminum divider separated the apparatus into halves $(118 \times 21.8 \times 29 \mathrm{~cm})$. Therefore, boundaries existed which marked off four equal areas $(59 \times 21.8 \mathrm{~cm})$.

Procedure. Each rat was confined to one half of the apparatus for $1 \mathrm{~h}$. The rat was then returned to its home cage for $5 \mathrm{~min}$. During this time, the divider separating the two halves of the apparatus was removed. Next, the rat was placed back into the apparatus on the habituated or familiar side. After $20 \mathrm{sec}$ had elapsed, the number of areas entered was recorded for each $5 \mathrm{sec}$ of the subsequent 10 -min test session (activity measure). Also, the location of the animal on the familiar or the novel half of the apparatus was recorded at the end of each 5 -sec time sample (exploration measure). Therefore an animal could score high on the activity measure and low on the exploration measure, or vice versa.

Testing order of subjects alternated between isolation- and group-reared rats. Half of the animals within each rearing condition were confined to the right half of the apparatus, and half to the left half. The box was sprayed with a mild deodorant between animals to mask odors. Testing was conducted between 0900 and $1600 \mathrm{~h}$, in the light part of the animals' day-night cycle.

\section{Results}

The exploration measure used was novelty preference, defined as the frequency of being on the novel half of the apparatus. The activity measure used was the number of entries into the four quarters of the apparatus. Results of the experiments with male and female rats will be presented separately, since the experiment with male rats was run at another time as a partial replication of the experiment with the females.

Novelty preference. In both experiments, frequency of novelty preference was analyzed over five blocks of 2 min each with two-way analyses of variance. Both male and female rats reared in isolation showed significantly enhanced preference for novelty compared with controls $[F(1,18)=9.78,8.72$, respectively, both ps $<.01$, Figure 4]. The overall preference for novelty increased over time within the trial for male $[F(4,72)=2.49, p<.05]$, but not female $[F(4,72)=1.32, p>.05]$ rats. In neither case was there a significant interaction of rearing condition with time course of novelty preference.

Sixteen of the 20 male rats and 16 of the 20 female rats showed greater preference for the novel than for the familiar side. Based on the binomial test, both of these results are significant at the $p=.006$ level.

Activity. Frequencies of entries into the four quarters of the apparatus were analyzed over five 2-min time blocks with two-way analyses of variance. Isolation rearing did not affect the frequency of entries either for male [isolation-reared, mean $=55$, group-reared, mean $=48 ; F(1,10)=2.24$, $\mathrm{p}>.05$ ] or female [isolation-reared, mean $=61$, group-reared, mean $=55 ; \mathrm{F}(1,10)=0.57, \mathrm{p}>.05$ ]

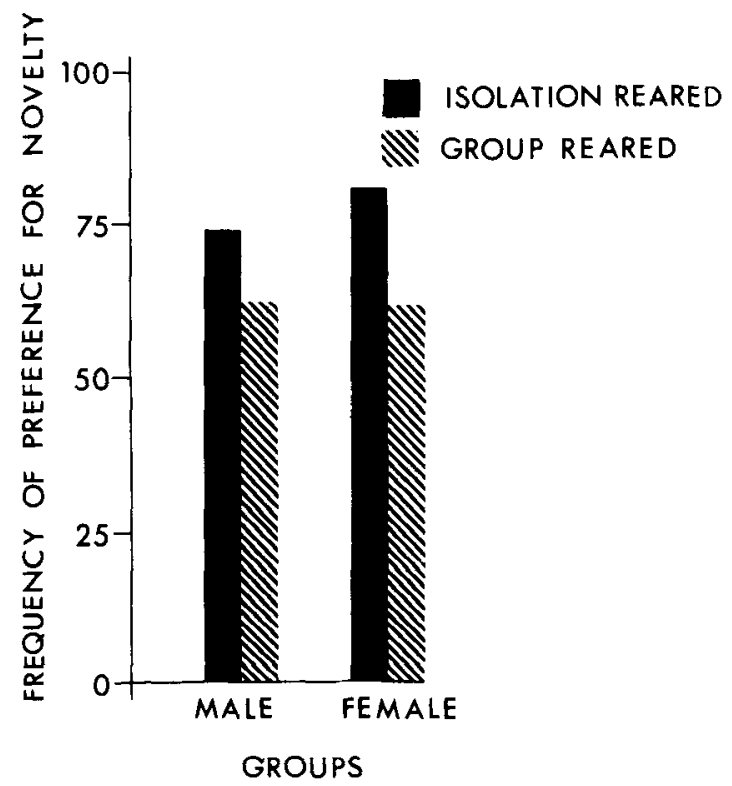

Figure 4. Mean frequency of preference for novelty of isolation-reared and group-reared rats in Experiment 2. 
rats. The frequencies of entries declined over time $[F(4,72)=33.62,61.02$ for males and females, respectively; both ps $<.001$ ], but rearing condition did not affect the rate of decline of entries.

\section{Discussion}

The main finding of Experiment 2 was that isolation-reared rats showed an enhanced preference for novel stimulation. This result supplements the finding of Ehrlich (1961) that rats reared in restricted environments showed higher levels of operant responding for stimulus change than controls. It also provides further support for the contention of Konrad and Melzack (1975) that isolation rearing exaggerates the normal response to novelty in a variety of species. In many of the studies that the latter authors reviewed, isolation rearing produces a diffuse emotional excitement in response to novel stimulation. However, since stimulus novelty also normally elicits exploratory behavior, it would be expected that isolation rearing would enhance exploratory behavior, as measured by novelty preference. This was demonstrated in the present study.

In Experiment 2, activity and exploration could be independently measured. The failure to find hyperactivity in isolates in the present experiment is possibly due to the relative insensitivity of the mode of measurement, compared with the measurement based on photocell beam interruptions of Experiment 1 . However, the lack of significant differences between the two groups suggests that the enhanced novelty preference of isolates was not due to higher activity levels. Other facts argue against the difference in novelty preference being due to an initial inertia or reluctance of the group-reared rats to explore the novel environment. Analysis of novelty preference over the course of the trial showed that the difference between the two groups was constant over time; that is, the enhanced novelty preference of isolates was not attributable to differences in the first portions of the test trial. In addition, the groupreared rats showed no obvious signs of "emotionality," such as defecation, freezing, or crouching, which might have interfered with exploratory tendencies.

Comparing these results with those of Experiment 1 , it is clear that the two measures of exploration provide different results. In a situation such as that used in Experiment 2, isolates are more exploratory. Apparently, isolates explore more in a situation in which exploration and locomotor activity are not incompatible forms of behavior. However, in Experiment 1 , the isolates had less opportunity to display increased exploration, since the stimulus object was located in a highly confined space, which was incompatible with the high levels of locomotor activity shown by these animals. It is also possible that the competing hyperkinesia of the isolates disrupts the performance of fine exploratory movements, such as manipulation rather than simple contact with objects. Isolates are inferior to groupreared animals on measures of manipulatory, but not nonmanipulatory, contacts with objects (Einon \& Morgan, 1976).

It is premature to conclude that the apparently enhanced levels of exploration and locomotor activity shown by isolates result from changes in a single behavioral mechanism. There has been no evidence to link the elevated levels of novelty preference and activity with each other, or with other behavioral effects of isolation, such as the differential responsiveness to both stimulant and depressant drugs (Einon, Stewart, Atkinson, \& Morgan, 1976; Sahakian et al., 1975).

\section{REFERENCES}

Berlyne, D. E. Arousal and satiation of perceptual curiosity in the rat. Journal of Comparative and Physiological Psychology, 1955, 48, 238-246.

BerLyne, D. E. Conflict, arousal and curiosity. New York: McGraw-Hill, 1960.

Dyne, L. J., \& HuGHEs, R. N. Effects of methylphenidate on activity and reaction to novelty in rats. Psychonomic Science, 1970, 19, 267-268.

EhRLICH, A. Effects of past experience on exploratory behaviour in rats. Canadian Joumal of Psychology, 1961, 15, 15-19.

Einon, D. F., \& Morgan, M. J. Habituation of object contact in socially-reared and isolated rats (Rattus Norvegicus). Animal Behaviour, 1976, 24, 415-420.

Einon, D. F., Morgan, M. J., \& Sahakian, B. J. The development of intersession habituation and emergence in socially reared and isolated rats. Developmental Psychobiology, 1976, 8, 553-560.

Einon, D. F., Stewart, J., Atrinson, S., \& Morgan, M. J. Effects of isolation on barbiturate anaesthesia in the rat. Psychopharmacologia (Berl.) 1976, 50, 85-88.

KonRAD, K., \& MELzack, R. Novelty-enhancement effects associated with early sensory-social isolation. In A. Riesen (Ed.), The developmental neuropsychology of sensory deprivation. London: Academic Press, 1975. Pp. 253-276.

LeYland, M., Robins, T. W., \& Iversen, S. D. Locomotor activity and exploration: The use of traditional manipulators to dissociate these two behaviors in the rat. Animal Learning \& Behavior, 1976, 4, 261-265.

LORE, R. K., \& Levowitz, A. Differential rearing and free versus forced exploration. Psychonomic Science, 1966, 5, 421-422.

Luchins, A. S., \& Forgus, R. H. The effects of differential postweaning environment on the rigidity of an animal's behavior. Journal of Genetic Psychology, 1955, 86, 51-58.

Mandell, A. J., Segal, D. S., Kuczensku, R. T., \& Knapp, S. Amphetamine-induced changes in the regulation of neurotransmitter biosynthetic and receptor functions in the brain. In: Pharmacology and the future of man. Proceedings of the 5th International Congress of Pharmacology, San Francisco, 1972. Basel: Karger, 1973. Pp. 95-105.

MoRGAN, M. J. Effects of post-weaning environment on learning in the rat. Animal Behaviour, 1973, 21, 429-442.

Robrins, T. W., \& Iversen, S. D. A dissociation of the effects of d-amphetamine on locomotor activity and exploration in rats. Psychopharmacologia (Berl.), 1973, 28, 155-164.

RosenzWEIG, M. R. Effects of environment on development of brain and of behavior. In E. Tobach, L. R. Aronson, \& E. Shaw 
(Eds.), The biopsychology of development. New York: Academic Press, 1971. Pp. 303-342.

Sahakian, B. J., Robbins, T. W., Morgan, M. J., \& Iversen, S. D. The effects of psychomotor stimulants on stereotypy and locomotor activity in socially-deprived and control rats. Brain Research, 1975, 84, 195-205.

SChNeIder, J., \& Gross, C. G. Curiosity in the hamster. Journal of Comparative and Physiological Psychology, 1965, 59, 150-152.

Syme, L. A. Social isolation at weaning: Some effects on two measures of activity. Animal Learning \& Behavior, 1973, 1, 161-163.

Valzelli, L. The "isolation syndrome" in mice. Psychopharmacologia (Berl.), 1973, 31, 305-320.

WELKER, W. I. Free vs. forced exploration of a novel situation by rats. Psychological Reports, 1957, 3, 95-108.

(Received for publication November 5, 1976; revision accepted February 10, 1977.) 